\title{
Sistema Integrado para Cancelamento de Realimentação e Redução do Efeito de Oclusão em Aparelhos Auditivos
}

\author{
Renata Coelho Borges e Márcio Holsbach Costa
}

\begin{abstract}
Resumo - Este trabalho apresenta uma estratégia conjunta para cancelamento de realimentação e redução do efeito de oclusão em aparelhos auditivos. A realimentação positiva e o efeito de oclusão são fenômenos indesejados, nos quais, em geral, esforços para a atenuação de um correspondem à ampliação do outro. De forma a evitar esse antagonismo, propõe-se uma abordagem ativa baseada no algoritmo adaptativo Least Mean Square com atualização dos coeficientes no domínio da frequência, no qual o efeito de oclusão é tratado em baixas frequências e o de realimentação em altas. Simulações computacionais e avaliação psicoacústica com voluntários suportam a eficácia do método proposto.
\end{abstract}

Palavras-Chave- Aparelho auditivo, realimentação, efeito de oclusão.

Abstract - This work presents a joint strategy for feedback canceling and reduction of the occlusion-effect in hearing aids. Positive feedback and the occlusion-effect are undesired phenomena, in which, in general, efforts for attenuation of one correspond to amplification of the other. To avoid this antagonism, an active approach based on the Least Mean Square algorithm with updates in the frequency domain is proposed; in which, the occlusion effect is handled in low frequencies, while the feedback is treated in high frequencies. Computational simulations and psychoacoustic assessment with volunteers support the efficiency of the proposed method.

Keywords - Hearing aids, feedback, occlusion effect.

\section{INTRODUÇ̃̃o}

A perda auditiva é a deficiência sensorial mais comum, afetando cerca de 5\% da população mundial e acarretando importantes consequências nos aspectos social e econômico.

A maior parte dos indivíduos com perda auditiva incapacitante vive em países de baixa e média renda e, para muitos, aparelhos auditivos são dispositivos cruciais para a reintegração social e econômica. No entanto, atualmente, a produção de aparelhos auditivos atende a apenas $10 \%$ da necessidade global e a maioria dos dispositivos é projetada, fabricada e distribuída em economias industrializadas [1]. Segundo a Organização Mundial da Saúde, aparelhos auditivos fornecidos aos países emergentes precisam ser robustos e de baixa manutenção, ser eficientes em termos do uso de energia e incluir sistemas de compressão, redução de ruído e cancelamento de realimentação [1].

A realimentação acústica tem sido citada em inúmeras pesquisas de mercado como um fenômeno causador de grande

Renata Borges, Departamento Acadêmico de Eng. Elétrica, Universidade Tecnológica Federal do Paraná, Cornélio Procópio - PR, e-mail renatacoelho@ utfpr.edu.br. Márcio Costa, Depto de Eng. Elétrica e Eletrônica - SC, Universidade Federal de Santa Catarina - SC, Florianópolis, e-mail: costa@eel.ufsc.br Este trabalho foi parcialmente financiado pelo CNPq (315020/2018-0). insatisfação aos usuários de aparelhos auditivos. Ela ocorre devido ao acoplamento acústico entre alto-falante e microfone, resultando em uma distorção no sinal enviado ao usuário. Com o aumento da amplificação essa distorção é percebida como um apito de alta intensidade, podendo levar o sistema à instabilidade. Esse problema é mais pronunciado em equipamentos do tipo completamente aberto (open fit) ou com abertura de ventilação ampla [2]. Dessa forma, a amplificação máxima do sinal captado pelo microfone tem de ser limitada. Com o fechamento parcial ou total da abertura de ventilação, a realimentação diminui e o fator de ganho pode ser aumentado. Entretanto, nessa situação, ocorre a sensação de "canal auditivo fechado" e o efeito de oclusão se pronuncia.

$\mathrm{O}$ efeito de oclusão é causado pelo aumento de energia acústica no interior do canal auditivo [3,4]. Quando o usuário do aparelho auditivo fala ou mastiga são produzidas vibrações nas porções cartilaginosas do canal auditivo, provocando aumento significativo de potência em baixas frequências (que não podem ser dissipadas em função do fechamento do canal).

Em [5] foi apresentada uma análise do funcionamento concorrente de sistemas individuais de cancelamento de realimentação e de redução do efeito de oclusão. Como resultado, verificou-se que esses sistemas não apresentam interação significativa em função de operarem em bandas distintas de frequência, uma vez que o efeito de oclusão ocorre preponderantemente para frequências inferiores a $500 \mathrm{~Hz}$, enquanto que a realimentação é mais comum acima de $1 \mathrm{kHz}$. A partir do exposto e dada a necessidade de minimização da complexidade computacional dos algoritmos embarcados em aparelhos auditivos (em função de restrições práticas de tamanho e limitação computacional) encontra-se motivação para a proposição de um sistema integrado para redução do efeito de oclusão e cancelamento de realimentação.

Vários esforços na integração de subsistemas de aparelhos auditivos têm sido apresentados na literatura, como por exemplo: redução de ruído e compressão dinâmica [6]; e redução de ruído e controle ativo de ruído [7]. No conhecimento dos autores, até o momento, não foi apresentada nenhuma proposta ou estudo envolvendo a integração dos sistemas de cancelamento de realimentação e de redução de oclusão.

Neste trabalho é apresentada uma proposta de integração dos sistemas de cancelamento de realimentação e de redução do efeito de oclusão baseada no filtro adaptativo Least Mean Square (LMS), na qual, o processo de filtragem é realizado no domínio do tempo e a atualização dos coeficientes no domínio da frequência. Simulações e um experimento psicoacústico com voluntários suportam a funcionalidade da abordagem. 


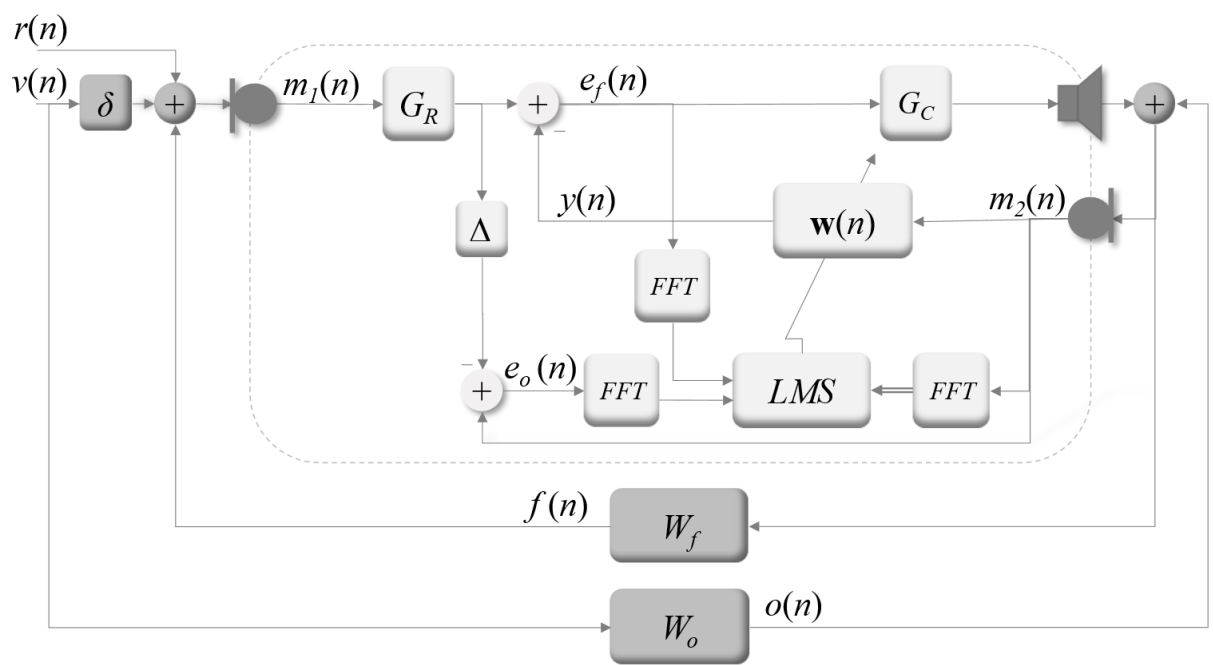

Fig. 1 - Sistema de cancelamento de realimentação e oclusão.

A seguir, a seção II apresenta a proposta do sistema. $\mathrm{Na}$ seção III são descritas as simulações e experimento psicoacústico realizados para avaliar o desempenho da proposta realizada, enquanto que na seção IV são apresentados os resultados e discussão. A seção $\mathrm{V}$ apresenta as conclusões do trabalho. Ao longo deste texto, variáveis escalares são definidas por letras minúsculas em itálico, constantes por letras maiúsculas em itálico, vetores por letras minúsculas em negrito e matrizes por letras maiúsculas em negrito.

\section{Sistema Proposto}

A estrutura conjunta de cancelamento de realimentação e redução do efeito de oclusão é apresentada na Fig. 1. Diferentemente da ação concorrente de dois sistemas individuais, como apresentado em [5], é utilizado apenas um filtro adaptativo representado por $\mathbf{w}(n)$.

Como base de implementação é utilizada a estrutura realimentada de cancelamento de oclusão proposta em [8], na qual o sinal de um microfone posicionado internamente ao molde acoplado ao canal condutivo do usuário fornece o sinal de referência para o cancelador realimentado.

O processamento dos sinais é feito no domínio do tempo, mas a adaptação é realizada no domínio da frequência [9]. Isso é necessário para evitar atrasos no caminho direto e, portanto, a degradação do processo de redução do efeito de oclusão.

Assume-se que o alto-falante e os dois microfones possuem resposta plana em frequência na faixa de interesse. Caso isso não seja verdade, podem ser utilizados equalizadores para a compensação.

\section{A. Estrutura de Aquisição e Processamento de sinais}

A fala do usuário do aparelho auditivo é representado por $v(n)$, sendo $\delta$ o atraso de propagação entre o sistema fonador e o microfone externo. Sinais acústicos externos, como a fala de outras pessoas e ruído ambiental, são representados por $r(n)$; $m_{1}(n)$ é o sinal captado pelo microfone externo, que é imediatamente processado pelo sistema $G_{R}$ de redução de ruído e compensação em frequência; $e_{f}(n)$ é o sinal enviado ao altofalante antes de ser processado por $G_{C}$, o qual é associado ao processo de compressão dinâmica; $m_{2}(n)$ é o sinal captado por um microfone auxiliar posicionado internamente ao canal auditivo. O sinal de cancelamento $y(n)$ é a saída do filtro adaptativo de resposta finita ao impulso, cujos coeficientes são dados por $\mathbf{w}(n)=\left[w_{0}(n) w_{1}(n) \ldots w_{N-1}(n)\right]^{\mathrm{T}}$, em que $N$ é o número de coeficientes do filtro adaptativo. O parâmetro $\Delta$ é o atraso de controle estimado, devido ao processo de conversão digital-analógico, amplificadores de potência e alto-falante.

Os blocos $W_{f}$ e $W_{o}$ representam os sistemas de realimentação e oclusão, resultando, respectivamente, nos sinais $f(n)$ e $o(n)$. Os sinais $e_{o}(n)$ e $e_{f}(n)$ carregam informação relativa à distorção associada, respectivamente, ao sinal de realimentação e ao efeito de oclusão. O sinal de erro de oclusão, $e_{o}(n)$, é calculado pela diferença entre o sinal de fala atrasado e o sinal atual no interior do canal auditivo. $\mathrm{O}$ sinal de erro de realimentação, $e_{f}(n)$, é dado pela diferença entre o sinal de saída do cancelador $\mathbf{w}(n)$ e o sinal proveniente do microfone externo.

\section{B. LMS com Atualização no Domínio da Frequência}

O algoritmo de bloco LMS no domínio da frequência é amplamente utilizado com o objetivo de melhorar a eficiência computacional e a taxa de convergência em relação à sua implementação convencional no domínio do tempo [10]. Entretanto, o LMS no domínio da frequência resulta em um atraso de um bloco de dados entre a entrada e a saída do filtro. Em aplicações de controle, como é o caso da redução do efeito de oclusão, esse atraso degrada o desempenho do sistema. Portanto, uma alternativa é a realização do processo de adaptação no domínio da frequência e do processo de filtragem no domínio do tempo [9]. Dessa forma, não são introduzidos atrasos de processamento no caminho direto.

O algoritmo LMS no domínio da frequência minimiza a seguinte função custo:

$$
J=\mathbf{e}^{\mathrm{T}}(n) \mathbf{e}(n)=\sum_{i=0}^{N-1} e^{2}(n-i),
$$

que é uma estimativa da média de bloco da função custo $J=E\left[\mathbf{e}^{\mathrm{T}}(n) \mathbf{e}(n)\right]$, em que $\mathbf{e}(n)$ é um vetor de erro a ser definido posteriormente; apresentando propriedades de convergência semelhantes às do algoritmo LMS, que minimiza uma estimativa instantânea da função custo $J=E\left[e^{2}(n)\right]$ [3].

A equação de atualização do filtro adaptativo no domínio do tempo é dada por

$$
\mathbf{w}(n+1)=\mathbf{w}(n)+\mu \cdot \operatorname{IFFT}\left\{\mathbf{m}_{2}^{*}(k) \mathbf{e}(k)\right\}_{+},
$$


em que $\mu$ é o coeficiente de convergência; o operador $\left\{^{*}\right\}$ denota o complexo conjugado; IFFT $\{\cdot\}$ é a transformada inversa de Fourier; e $\{\cdot\}+$ refere-se à parte causal do argumento, sendo equivalente à restrição de causalidade do gradiente [11]. Assume-se um bloco de tamanho de $N$ novas amostras para que o filtro adaptativo seja atualizado. A transformada de Fourier (FFT) é calculada para blocos de tamanho $2 N$ para evitar efeitos de convolução circular [11].

Os vetores $\mathbf{m}_{2}(k)$ e $\mathbf{e}(k)$, definidos no domínio da frequência, possuem tamanho $2 N$, e são calculados a partir da FFT dos respectivos sinais no domínio do tempo. O vetor $\mathbf{m}_{2}(k)$ é calculado por

$$
\mathbf{m}_{2}(k)=F F T\left\{\left[\mathbf{m}_{2}^{\mathrm{T}}(n) \mathbf{m}_{2}^{\mathrm{T}}(n-N)\right]^{\mathrm{T}}\right\},
$$

em que $\mathbf{m}_{2}(n)=\left[m_{2}(n) m_{2}(n-1) \ldots m_{2}(n-N+1)\right]^{\mathrm{T}}$. O vetor $\mathbf{e}(k)$ é formado a partir de uma combinação dos sinais de erro de realimentação, $e_{f}(n)$, e erro de oclusão, $e_{0}(n)$, calculados, respectivamente, por

$$
\begin{gathered}
e_{\mathrm{o}}(n)=m_{2}(n)-m_{1}(n-\Delta) G, \\
e_{f}(n)=m_{1}(n) G_{R}-y(n),
\end{gathered}
$$

em que $G$ é o ganho total decorrente das rotinas $G_{R}$ e $G_{C}$. As respectivas transformadas de Fourier são calculadas por

$$
\begin{aligned}
& \mathbf{e}_{o}(k)=F F T\left\{\left[\mathbf{0} \mathbf{e}_{o}^{\mathrm{T}}(n)\right]^{\mathrm{T}}\right\}, \\
& \mathbf{e}_{f}(k)=F F T\left\{\left[\mathbf{0} \mathbf{e}_{f}^{\mathrm{T}}(n)\right]^{\mathrm{T}}\right\},
\end{aligned}
$$

em que 0 é um vetor de dimensão $N \times 1$, preenchido por zeros, usado para garantir correlação na atualização do filtro adaptativo [9]; $\mathbf{e}_{o}(n)=\left[\begin{array}{llll}e_{o}(n) & e_{o}(n-1) & \ldots & e_{o}(n-N+1)\end{array}\right]^{\mathrm{T}} ; \quad \mathrm{e}$ $\mathbf{e}_{f}(n)=\left[e_{f}(n) e_{f}(n-1) \ldots e_{f}(n-N+1)\right]^{\mathrm{T}}$. Dessa forma, o vetor erro utilizado para adaptar o filtro adaptativo é uma combinação dos bins resultantes da transformada de Fourier de $\mathbf{e}(n)=\left[\mathbf{e}_{o}{ }^{\mathrm{T}}(n)\right.$ $\left.\mathbf{e}_{f}^{\mathrm{T}}(n)\right]^{\mathrm{T}}$, de forma que:

$$
\begin{aligned}
\mathbf{e}(k)= & {\left[\mathrm{e}_{o}(-N) \mathrm{e}_{o}(-N+1) \ldots \mathrm{e}_{o}\left(-N-k_{1}\right)\right.} \\
& \mathrm{e}_{f}\left(-N-k_{1}-1\right) \ldots \mathrm{e}_{f}\left(N-k_{1}\right) \\
& \left.\mathrm{e}_{o}\left(N-k_{1}+1\right) \mathrm{e}_{o}\left(N-k_{1}+2\right) \ldots \mathrm{e}_{o}(N-1)\right]^{\mathrm{T}}
\end{aligned}
$$

em que $k_{1}$ é o índice correspondente ao limite de separação entre a faixa de frequências dominantes da realimentação e oclusão. Para índices $k>k_{1}$ a atualização dos coeficientes é realizada com o objetivo de identificar a resposta em frequência do sistema de realimentação, enquanto que para $k \leq k_{1}$ o objetivo é realizar a redução (cancelamento) do efeito de oclusão.

\section{MATERIAIS E MÉTODOS}

Esta seção apresenta as configurações dos experimentos realizados para demonstrar a funcionalidade e o desempenho do sistema proposto.

Foram utilizadas respostas ao impulso obtidas em processos de identificação realizados em situações reais de operação de aparelhos auditivos [13]. A resposta ao impulso do efeito de realimentação corresponde ao caso de uma abertura de ventilação do molde do aparelho auditivo com $3 \mathrm{~mm}$ de diâmetro, e a resposta ao impulso associada ao efeito de oclusão está relacionada ao fechamento completo do molde (Fig. 2).

A frequência de amostragem foi definida em $16 \mathrm{kHz}$. Com o objetivo de simplificar o processamento realizado pelo aparelho auditivo estabeleceu-se $G_{R} \equiv q^{-16}$ (em que $q$ é o operador atraso), equivalente a um atraso de processamento de
$1 \mathrm{~ms}$ (sendo que o atraso máximo tolerável para este tipo de aplicação é de cerca de 8 a 10 ms [2]). O sistema de compressão e compensação da resposta em frequência foi definido como um fator de amplificação simples. Os demais parâmetros utilizados foram: $\Delta=1$ e $\delta=14$ [14]; $N=150 ; k_{1}=11$ (correspondendo a $550 \mathrm{~Hz}) ; \mathbf{w}(0)=\left[\begin{array}{llll}0 & 0 & \ldots & 0\end{array}\right]^{\mathrm{T}}$. Assumiu-se que o ruído ambiental é desprezível. As simulações foram realizadas no ambiente $\mathrm{MatLab}^{\circledR}$.

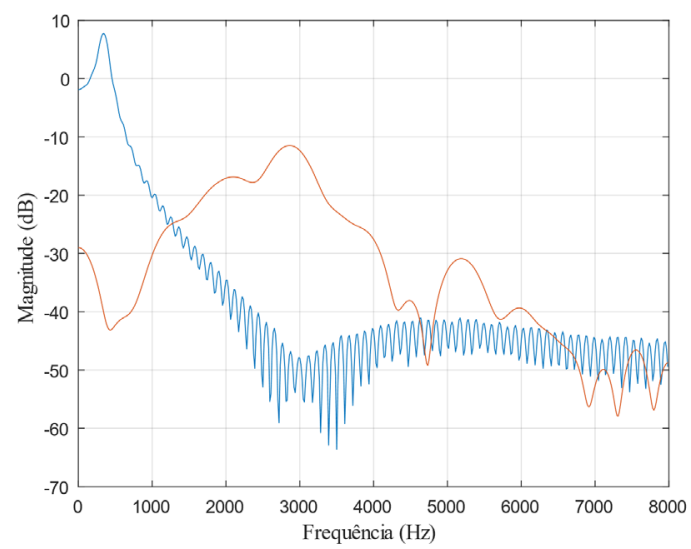

Fig. 2 - Resposta em frequência dos sistemas de oclusão (assumindo fechamento completo do canal auditivo) (azul) e realimentação (assumindo uma abertura de ventilação de $3 \mathrm{~mm}$ ) (vermelho).

\section{A. Simulações com Sinais Artificiais}

Nesta subseção são realizadas simulações com sinais artificiais para verificar a estabilidade e convergência do processo de adaptação do cancelador proposto. Duas situações foram abordadas. No primeiro caso, a fala do usuário $v(n)$ foi simulada a partir da filtragem de um ruído branco gaussiano por um modelo autorregressivo (ordem 21, $\sigma_{\mathrm{v}}^{2}=3,5 \times 10^{-4}$ ) de forma a apresentar as características de um som vozeado [14] (situação em que ocorre o efeito de oclusão). No segundo caso, foi utilizado ruído branco (sem processamento), para simular um som não vozeado $v(n)$. As simulações de Monte Carlo foram realizadas calculando a média de 10 realizações. Os parâmetros do sistema foram $\mu=10^{-5}$ (escolhido como $75 \%$ do passo para o qual o sistema instabiliza) e $G_{C}=4(12 \mathrm{~dB})$.

\section{B. Critérios Objetivos}

O desempenho do sistema proposto foi avaliado por meio de três critérios objetivos de qualidade de fala: (a) short-time objective intelligibility (STOI); (b) cepstrum distance (CEP); e (c) perceptual evaluation of speech quality (PESQ). O STOI estima a inteligibilidade da fala, resultando em um valor entre 0 e 1. O CEP é uma medida de distância que fornece uma estimativa do erro log-espectral entre dois sinais; e o PESQ prioriza aspectos associados à qualidade perceptual, podendo ser relacionado ao critério mean opinion score (MOS).

As métricas selecionadas foram aplicadas a uma fala humana $v(n)$, sexo masculino, extraída de [15] para 4 cenários diferentes: (a) sistema sem influência de realimentação e oclusão; (b) sistema sujeito à influência da realimentação; (c) sistema sujeito à influência do efeito de oclusão; (d) sistema sujeito à influência da realimentação e da oclusão. Para cada um desses cenários, assumiu-se duas condições de atualização dos coeficientes do filtro adaptativo: "desligada" (sem cancelador) 
ou "ligada" (com cancelador). Os parâmetros utilizados foram: $\mu=10^{-3}$ e $G_{C}=4(12 \mathrm{~dB})$. O ganho escolhido resulta em microfonia quando o cancelador não é acionado.

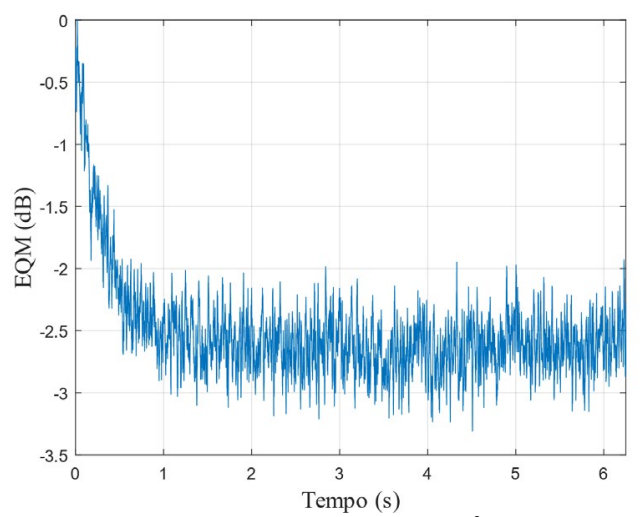

Fig. 3 - Evolução do erro quadrático médio $E\left[e_{\mathrm{o}}^{2}(n)\right]$ para o sinal vozeado simulado.

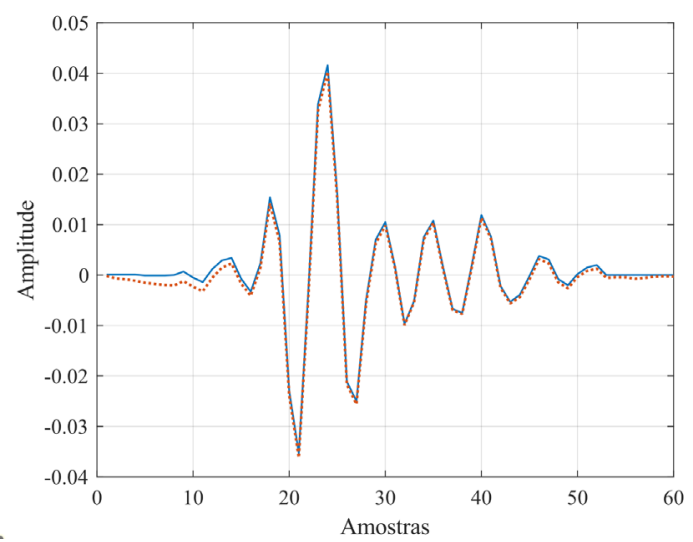

Fig. 4 - Valor médio dos coeficientes do filtro adaptativo (vermelho) e resposta ao impulso do sistema de realimentação (azul).

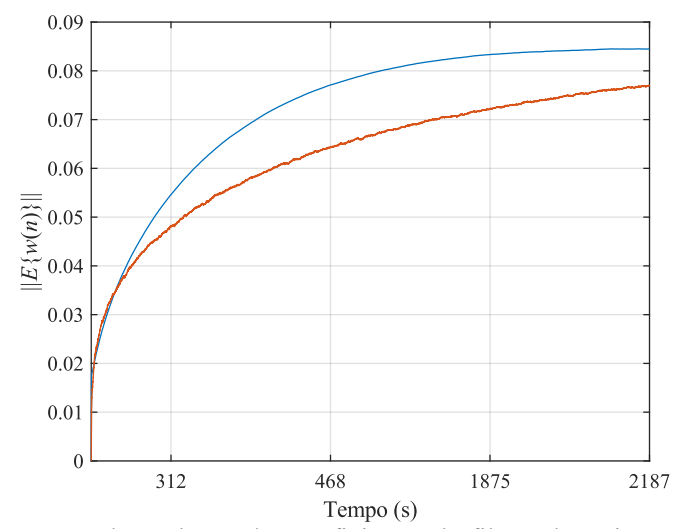

Fig. 5 - Norma da evolução dos coeficientes do filtro adaptativo para a sinais não vozeados (azul) e sinais vozeados (vermelho).

\section{Experimento Psicoacústico}

Nesta subseção é apresentada a metodologia utilizada para a avaliação subjetiva dos sinais.

Um conjunto de cinco sentenças obtidas de [15], com duração entre 3 e 6 segundos, foi utilizada como $v(n)$ na Fig. 1. Foram investigados os mesmos 4 cenários e 2 condições de atualização dos coeficientes descritos na seção III.B. De modo a evitar que o sistema instabilizasse, causando microfonia, o ganho $G_{C}$ foi reduzido para 9,5 dB. O passo de adaptação utilizado foi $\mu=10^{-3}$, escolhido como $75 \%$ do passo para o qual o sistema instabiliza.

Os arquivos de áudio resultantes foram gravados e avaliados posteriormente por dez voluntários, sem histórico ou reclamações de problemas auditivos, utilizando fones de ouvido. Solicitou-se a impressão pessoal sobre a qualidade da fala e conforto acústico da condição "atualização ligada" em relação à condição "atualização desligada" para os quatro cenários avaliados. A escala de pontuação foi definida por números inteiros variando entre -3 (muito ruim) e 3 (muito bom). Pontuação positiva valoriza o desempenho do sistema proposto.

\section{Resultados E Discussão}

Esta seção apresenta os resultados obtidos a partir dos experimentos descritos na seção III.

\section{A. Simulações com Sinais Artificiais}

A Fig. 3 apresenta a evolução do erro quadrático médio do erro de oclusão $\mathrm{E}\left[e_{\mathrm{o}}^{2}(n)\right]$, indicando uma redução média de 2,5 $\mathrm{dB}$ em regime permanente para o caso de fala vozeada.

Assumindo-se o caso de fala não vozeada, o valor médio do vetor de coeficientes em regime permanente é apresentado na Fig. 4, em vermelho. Verifica-se que, a despeito de algumas pequenas distorções nas amostras iniciais, o filtro adaptativo foi capaz de identificar adequadamente a resposta ao impulso do sistema de realimentação (em azul).

Na Fig. 5 é apresentada a norma $\mathrm{L}_{2}$ da evolução do vetor de coeficientes, $\|E[\mathbf{w}(n)]\|_{2}$ para ambos os casos. Em vermelho se encontra o comportamento da norma dos coeficientes para sinais vozeados (situação que evidencia o efeito de oclusão) e em azul para sinais não vozeados. Em ambas as situações se verifica convergência dos coeficientes, que se apresenta bastante lenta em decorrência do valor do passo de adaptação do filtro, escolhido de forma a obter-se uma clara visualização do comportamento de adaptação. Os resultados apresentados evidenciam a funcionalidade do método proposto em condições controladas.

TABELA I. CRITÉRIOS OBJETIVOS.

\begin{tabular}{clccc}
\hline Tipo de distorção & Cancelador & STOI & CEP & PESQ \\
\hline \hline \multirow{2}{*}{ Nenhuma } & Desligado & 1,00 & 0 & 4,64 \\
& Ligado & 1,00 & 0,05 & 4,64 \\
\hline Somente & Desligado & 0,99 & 0,28 & 4,43 \\
Oclusão & Ligado & 0,98 & 0,37 & 3,85 \\
\hline Somente & Desligado & 0,38 & 7,03 & 1,18 \\
Realimentação & Ligado & 0,99 & 0,33 & 4,14 \\
\hline Oclusão e & Desligado & 0,38 & 7,03 & 1,18 \\
Realimentação & Ligado & 0,95 & 0,61 & 3,27 \\
\hline
\end{tabular}

\section{B. Critérios Objetivos}

A TABELA I apresenta os resultados obtidos para os 4 cenários investigados e 2 condições do filtro adaptativo, em termos dos critérios objetivos escolhidos. Em condições normais (sem distorção) o cancelador proposto resulta em índices semelhantes aos da fala não processada (ou seja, não introduz distorções). No caso de existência de microfonia (situações de "somente realimentação" e "oclusão e realimentação" com cancelador desligado) os índices de 
qualidade e inteligibilidade indicam forte distorção do sinal não processado. Por outro lado, o acionamento do cancelador proposto controla a realimentação, resultando em melhor qualidade e inteligibilidade da fala. Finalmente, no caso de "somente oclusão" verifica-se que o cancelador diminui a qualidade da fala, isso decorre, provavelmente, da redução de potência em baixas frequências. Note-se, entretanto, a inexistência de critérios objetivos para quantificação do efeito da oclusão.

\section{Experimento Psicoacústico}

Os resultados obtidos no experimento psicoacústico são apresentados na Fig. 6.

No primeiro experimento (Fig. 6a) é feita uma comparação do efeito do cancelador na qualidade da fala para o caso sem influência dos sistemas de realimentação e oclusão. Nessa situação, 94\% dos participantes relataram aproximadamente a mesma qualidade (com e sem cancelador). O segundo experimento (Fig. 6b) trata da situação "somente oclusão", na qual $94 \%$ dos voluntários indicam melhor qualidade do sinal processado. Para o caso "somente realimentação" (Fig. 6c), $92 \%$ dos voluntários relataram semelhança na qualidade da fala processada e não processada. Isto era esperado, em função da inexistência de microfonia nos sinais originais. No caso "realimentação e oclusão" (Fig. 6d), 60\% dos voluntários indicaram a preferência pelo sinal processado pela estrutura proposta. Os $40 \%$ restantes relataram uma sensação de metalização do som e a persistência de abafamento. Esse efeito pode estar relacionado ao passo de convergência utilizado, exigindo um critério de projeto mais específico. Por outro lado, as respostas impulsivas utilizadas são associadas aos respectivos piores casos. Em situações reais, as distorções não são tão extremas [16]. Em nenhum dos casos foi relatada diminuição da inteligibilidade da fala.

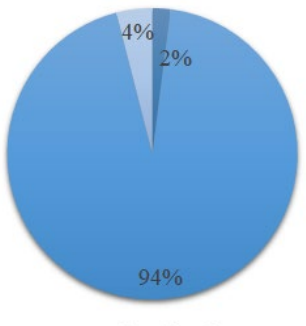

(a)

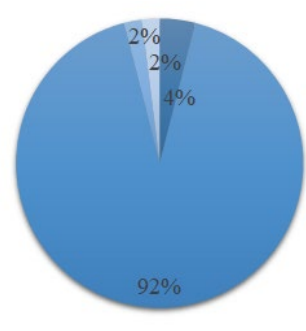

$\square-1=0=1=2$

(c)

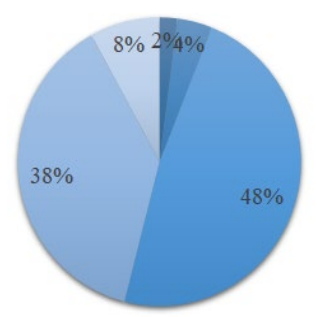

แ-1 $-0=1 \backsim 2 \backsim 3$

(b)

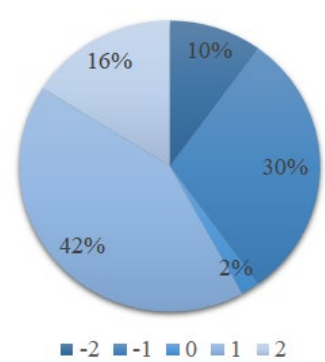

(d)
Fig. 6 - Avaliação psicoacústica para os casos cancelador ligado e desligado nos seguintes cenários: (a) sistema sem distorção; (b) sistema de oclusão; (c) sistema de realimentação; e (d) sistemas de oclusão e realimentação. Pontuação positiva favorece o cancelador proposto.

\section{CONCLUSÕES}

Este trabalho apresentou a proposta de um cancelador adaptativo integrado para redução conjunta do efeito de oclusão e realimentação em aparelhos auditivos. Resultados de simulação demonstram a funcionalidade do sistema proposto em termos de capacidade de rastreamento, controle da realimentação e estabilidade do sistema. Experimentos psicoacústicos indicam maior satisfação de voluntários com o sistema proposto em todos os cenários avaliados. Entretanto, verifica-se a pertinência de um estudo mais aprofundado em relação à definição dos parâmetros de projeto de forma a possibilitar a otimização do desempenho do sistema. A proposta de arquitetura conjunta para um sistema de redução dos efeitos de oclusão e realimentação tem como vantagem a economia de recursos computacionais.

\section{REFERÊNCIAS}

[1] World Health Organization, "Preferred profile for hearing-aid technology suitable for low- and middle-income countries", Technical Report, 2017.

[2] K. Chung, "Challenges and recent developments in hearing aids - Part II. Feedback and occlusion effect reduction strategies, Laser shell manufacturing processes, and other signal processing technologies", Trends in Amplification, v. 8, n. 4, pp. 125-164, 2004.

[3] J. Zwislocki, "Acoustical attenuation between the ears", The Journal of the Acoustical Society of America, v. 25, n. 4, pp. 752-759, 1953.

[4] J. Mejia, H. Dillon e M. Fisher, "Active cancellation of occlusion: an electronic vent for hearing aids and hearing protectors", The Journal of the Acoustical Society of America, v. 124, n. 1, pp. 235-240, 2008.

[5] R. C. Borges, W. D. Parreira e M. H. Costa, "Concurrent acoustical feedback and occlusion-effect cancellation in hearing aids: a simulationbased analysis", Congresso Brasileiro de Engenharia Biomédica, pp. 129-135, 2018.

[6] K. Ngo et al., "A combined multi-channel Wiener filter-based noise reduction and dynamic range compression in hearing aids", Signal Processing, v. 92, n. 2, pp. 417-426, 2012.

[7] R. Serizel et al., "Output SNR analysis of integrated active noise control and noise reduction in hearing aids under a single speech source scenario", Signal Processing, v. 91, n. 8, pp. 1719-1729, 2011.

[8] R. C. Borges, M. H. Costa, J. Cordioli e L. F. C. Assuiti, "Implementação e avaliação de um cancelador de oclusão adaptativo para aparelhos auditivos", Congresso Brasileiro de Engenharia Biomédica, pp. 1-5, 2012.

[9] B. Rafaely e S.J. Elliot, “A computationally efficient frequency-domain LMS algorithm with constraints on the adaptive filter", IEEE Transactions on Signal Processing, v. 48, n. 6, pp. 1649-1655, 2000.

[10] B. Farhang-Boroujeny e K. S. Chan. "Analysis of the frequency-domain block LMS algorithm”, IEEE Transactions on Signal Processing, v. 48, n. 8, pp. 2332-2342, 2000.

[11] J. J. Shynk, "Frequency-domain and multirate adaptive filtering", IEEE Signal Processing Magazine, v. 9, pp. 14-37, 1992.

[12] K. Chung, "Challenges and recent developments in hearing aids - Part I. Speech understanding in noise, microphone technologies and noise reduction algorithms", Trends in Amplification, v. 8, n. 4, pp. 83-124, 2004.

[13] R. C. Borges et al, "Impact of the vent size in the feedback-path and occlusion-effect in hearing aids", Biomedical Circuits and Systems Conference, pp. 1-4, 2014.

[14] R. C. Borges e M. H. Costa. "A feed forward adaptive canceller to reduce the occlusion effect in hearing aids", Computers in Biology and Medicine, v. 79, pp. 266-275, 2016.

[15] C. A. Ynoguti, Reconhecimento de Fala Contínua Usando Modelos Ocultos de Markov, Tese de Doutorado, Universidade Estadual de Campinas, Brazil, 1999.

[16] R. C. Borges et al., "Impact of the vent size in the feedback-path and occlusion-effect in hearing aids", IEEE Biomedical Circuits and Systems Conference, pp. 25-28, 2014. 